\title{
Identificación precoz e intervención breve en lesionados de tráfico con presencia de alcohol: primeros resultados
}

\author{
Alicia Rodríguez-Martos Dauer*, Elena Santamariña Rubio*, Xavier Martínez Gómez*, \\ Lluís Torralba Novella*, Mercè Escayola Coris**, Josep Martí Valls**, \\ Antoni Plasència Taradach**** \\ * Agència de Salut Pública (Barcelona) \\ * Hospital de Traumatologia i Rehabilitació Vall d'Hebron (Barcelona) \\ *** Universitat Autònoma de Barcelona \\ Enviar correspondencia a: \\ Alicia Rodríguez-Martos. Institut per a la Prevenció i Atenció a les Drogodependències. Agència de Salut Pública de Barcelona. \\ PI. Lesseps, 1. 08023-Barcelona. Tel. 932384560
}

\section{RESUMEN}

Objetivo: comprobar la efectividad de la intervención breve para reducir el consumo de alcohol en accidentados de tráfico con alcoholemia positiva, sin dependencia del alcohol.

Metodología: cribado de los lesionados de tráfico que acuden a urgencias de traumatología mediante un test de alcohol en saliva e intervención sobre los pacientes positivos. Los pacientes son evaluados y aleatoriamente distribuidos en grupos de Intervención Breve e Intervención Mínima. El seguimiento telefónico se hace al mes 3, 6 y 12.

Resultados: Hasta el 30 de Octubre de 2002, se incluyeron en el estudio 709 lesionados, habiéndose realizado la prueba del alcohol en 696. 93 pacientes dieron positivo $(13,4 \%$ ) y pudo intervenirse en 66 de ellos. El 50\% puntuaba positivo en el test AUDIT (puntuación media: 7,6). Los pacientes fueron distribuidos aleatoriamente entre intervención breve (30) e intervención mínima (36). El 77\% de pacientes tiene seguimiento.

El 79,4\% de los pacientes intervenidos ha reducido el consumo de alcohol, a los 3 meses y el 66,7\% \%, al año. Sobre todo disminuye el binge drinking (consumo copioso concentrado), el más peligroso para la conducción. A los 3 meses, ha reducido su consumo un porcentaje superior (en el límite de la significación estadística) de pacientes en el grupo de Intervención Breve. Al año, no se observan diferencias entre ambos grupos de intervención. La magnitud de la reducción es significativamente mayor en quienes tenían un resultado basal positivo en el test AUDIT.

Conclusiones: Aunque las muestras son homogéneas, es prematuro dar resultados de efectividad tanto por el tamaño aún reducido de los grupos estudiados, como porque todavía se incluyen pacientes y se están siguiendo los ya incluidos.

Palabras clave: lesionados, accidentes de tráfico, alcohol, cribado, intervención breve.

\section{SUMMARY}

Objective: to test the effectiveness of a brief intervention in reducing alcohol consumption in traffic casualties with positive blood alcohol concentration, and without alcohol dependency.

Methods: screening traffic casualties attending an emergency/trauma department, using an alcohol-saliva test, with intervention for positive patients. After assessment, patients are randomly allocated into either a brief or minimal intervention group. Phone follow-up takes place at month 3, 6,and 12.

Results: 709 traffic casualties had been included in the study by the end of October 2002. 696 patients had the alcohol test, which was positive in 93 cases (13.4\%). Intervention was possible with 66. Half of them had a positive AUDIT, with a mean score of 7.6. Patients were randomly allocated to brief intervention (30) and minimal intervention (36) groups. Followup has been made on $77 \%$.

$79.4 \%$ of patients who received an intervention had reduced alcohol consumption at month 3 , and $66.7 \%$ still had a reduced consumption at month 12 . Among consumption measures, binge drinking - the most dangerous for drivingshows the most important drop. At month 3, the percentage of patients who had reduced their intake was higher (at the limit of statistical significance) in the Brief Intervention group. At month 12, there are no apparent differences between the two intervention groups. The reduction in consumption is significantly greater among those who scored positive in the AUDIT at baseline.

Conclusion: Although the samples are homogeneous, it's too early to give effectiveness results, both because of the still small number of patients, and because we are still recruiting them and following-up those already enrolled.

Key words: casualties, traffic crashes, alcohol, screening, brief intervention.

Esta investigación está parcialmente subvencionada por el Fondo de Investigaciones Sanitarias (01/0903) y por el Servei Català de Trànsit. Ponencia presentada en las XXX JORNADAS NACIONALES DE SOCIDROGALCOHOL - Valladolid, 20,21 y 22 de Marzo de 2003 


\section{INTRODUCCIÓN}

Se estima que aproximadamente el $50 \%$ de las lesiones de tráfico fatales, así como el 20-30\% de las no fatales están relacionadas con el alcohol. Aunque legalmente se considera que un accidente de tráfico (AT) relacionado con el alcohol es aquél en el que un conductor o un peatón superan la alcoholemia legal en el momento del accidente', a partir de niveles de alcohol de 0,2 g/l, el rendimiento psicomotor queda afectado y el riesgo de colisión es significativo a partir de $0,5 \mathrm{~g} / \mathrm{l}$, siendo la probabilidad muy superior a la correspondiente a una alcoholemia inferior a 0,52. Tomar 50 más consumiciones de alcohol en una sola ocasión, dos o más veces al año dobla el riesgo de lesiones ${ }^{3}$, teniendo los bebedores más probabilidades de ingresar en urgencias por lesiones y el doble de posibilidades de sufrir complicaciones que los no bebedores ${ }^{4}$. Los pacientes que acuden a urgencias por lesiones tienen a su vez más probabilidades de estar bajo los efectos del alcohol que los no lesionados ${ }^{5}$.

En servicios de urgencias, la prevalencia de alcoholemias positivas entre lesionados de tránsito se sitúa entre la tercera o cuarta parte de los mismos, aunque se dan cifras que oscilan entre el $6 \%$ y el $33 \%{ }^{6}$, siendo los lesionados preferentemente jóvenes conductores que acuden en fin de semana ${ }^{7}$. Entre los pacientes admitidos por primera vez por un accidente de tráfico con presencia de alcohol, la probabilidad de ser readmitido en el mes subsiguiente por lesiones es 2,5 veces mayor que la correspondiente a un lesionado no intoxicado.

En España, el $41,2 \%$ de conductores y el $37,6 \%$ de los peatones fallecidos por AT en el año 2000 tenía una alcoholemia $>0,3 \mathrm{~g} / /^{9}$. Por lo que respecta a los lesionados atendidos por la policía en el mismo periodo, el $18,2 \%$ tenía alcoholemia positiva ${ }^{10}$, un porcentaje muy inferior al aportado por la policía municipal de Barcelona (46,8\%, en el año 2001). Si bien las últimas estimaciones apuntan a una menor prevalencia de alcoholemias positivas entre los implicados en AT, una tercera parte de los españoles sigue opinando que beber un par de combinados no representa un riesgo para la conducción"1".

Se estima ${ }^{12,13}$ que podría reducirse un tercio o más la incidencia de nuevos AT, si quienes conducen intoxicados se abstuvieran, y que los profesionales de urgencias podrían influir de forma importante en la toma concienciación acerca del riesgo del alcohol al volante y en la subsiguiente aceptación de medidas preventivas ${ }^{14}$.

La experiencia aversiva de un AT puede representar un "momento receptivo" 15 y el periodo postraumático representa una buena "ventana de oportunidad" para intervenciones educativas ${ }^{16}$. Se considera que los centros de traumatología se encuentran en una posi- ción privilegiada para cribar a los bebedores de riesgo y son un escenario adecuado para las intervenciones breves oportunistas ${ }^{17,18}$.

La eficacia de las intervenciones breves ha sido sólidamente demostrada, sobre todo en el ámbito de la atención primaria, por diversos estudios y metaanálisis $^{19-21}$. En el campo de la traumatología aparecen experiencias alentadoras, tanto en el sentido de una reducción del consumo ${ }^{22}$ no siempre significativa frente a controles ${ }^{23-25}$, como de una significativa reducción de la accidentalidad y de los reingresos ${ }^{22,24,25}$.

En España, la efectividad de las intervenciones breves sobre los bebedores de riesgo que acuden a atención primaria ha sido demostrada en diversos estudios, revisados en 2 recientes metaanálisis ${ }^{26,27}$. En el terreno de los lesionados de tráfico, éste es el primer estudio que se realiza en nuestro país.

El objetivo principal del estudio es verificar la efectividad de la intervención breve, comparada con la intervención mínima o consejo simple, para reducir el consumo de alcohol y, eventualmente, la accidentalidad relacionada con el mismo, en lesionados en AT con alcoholemia positiva y sin dependencia de alcohol, desde un centro de traumatología.

Los objetivos secundarios incluyen la descripción del perfil de los accidentados de tráfico con presencia de alcohol y verificar la utilidad de la intervención para derivar a tratamiento a los casos con posible dependencia.

Partimos de la hipótesis de que los lesionados en AT podrían reducir su consumo de alcohol o siquiera dejar de conducir bajo los efectos del alcohol tras una intervención breve motivacional, recibida tras haber sufrido la experiencia aversiva de un AT. La intervención breve motivacional debería ser, al cabo de un año, más efectiva que el simple consejo para la reducción del consumo de alcohol $y$, eventualmente, de nuevos problemas relacionados con el alcohol y la conducción.

\section{MATERIAL Y MÉTODOS}

Se eligió un diseño quasi-experimental, con cribado de alcohol en saliva de los AT y distribución aleatoria de los pacientes con resultado positivo $(\geq 0,2 \mathrm{~g} / \mathrm{l}$ de alcohol en sangre) entre intervención breve y mínima (grupo control). La ausencia de un auténtico grupo control (no intervención) obedece sobre todo a razones éticas. Por otra parte, la eficacia de la intervención breve ha sido suficientemente demostrada, reclamándose en la actualidad más estudios de efectividad que estudios controlados aleatorios. 
El estudio se realiza en el Hospital de Traumatología y Rehabiltación del complejo hospitalario Valle de Hebrón de Barcelona. A todo paciente lesionado de tráfico de 18 o más años y que se haya accidentado en las 6 horas precedentes se le hace una determinación de alcohol en saliva. A los que dan un resultado positivo (reactivo Alcohol-On-Site, de Roche Diagnostics, cualitativo con positividad a partir de $0,2 \mathrm{~g} / \mathrm{ll}$, se les ofrece una intervención. Los pacientes que aceptan son distribuidos aleatoriamente en grupo de intervención breve (IB) y grupo de intervención mínima (IM). Evaluación e intervención se hacen, una vez que el paciente está sobrio, en el servicio de urgencias o en la sala, si éste es ingresado.

La evaluación se hace mediante el cuestionario AUDIT ${ }^{28}$ (Test para la Identificación de los Trastornos por Uso de Alcohol) en versión española ${ }^{29}$, la Escala de Atribución de la Lesión ${ }^{30}$ y la Regleta del cambio ${ }^{31}$.

La intervención mínima (IM) consiste en un consejo simple, basado en el resultado de la evaluación, (aproximadamente $10^{\prime}$ ) y la entrega de material informativo, mientras la intervención breve (IB) consta de una intervención de tipo motivacional, que parte del resultado de la evaluación y acompaña al paciente en su reflexión acerca del cambio que podría beneficiarle (unos $20^{\prime}$ ), y de la entrega de material informativo y de autoayuda. Ambas intervenciones están pautadas en la correspondiente guía. Los pacientes con puntuación $\geq 15$ en el AUDIT, o que suman $\geq 4$ puntos en los ítems 4-6 que exploran dependencia, deben ser derivados para confirmación diagnóstica y eventual tratamiento. Estos pacientes son objeto de análisis diferenciado.

El seguimiento, ciego y por vía telefónica, se hace al mes 3, 6 y 12 preguntando el consumo a partir de las 3 primeras preguntas el AUDIT. Al año se pregunta también por la existencia de nuevos accidentes o incidentes en el tráfico.

\section{Análisis Estadístico}

Para el almacenamiento y seguimiento de los pacientes, se ha usado el procesador de datos ACCES 2000, y para el posterior análisis estadístico de los datos el paquete estadístico SPSS 10.0.

Se ha realizado un análisis descriptivo-comparativo de los datos obtenidos, incluyendo la prevalencia de alcoholemias positivas en la muestra global estudiada, la descripción de sus características y la identificación de los factores asociados a la presencia de alcohol. El análisis ha sido bivariado, mediante la prueba Chisquare o el Test exacto de Fisher, para las variables cualitativas. Para las variables cuantitativas se ha usado la prueba T-test para comparar las medias, y el Test $U$ de Mann-Whitney, como prueba no paramétrica, para comparar las medianas. El nivel de significación estadística usado ha sido $\mathrm{p}<0.05$.
Fig. 1. Algoritmo del estudio (diseño). Son elegibles para el estudio los lesionados en accidente de tráfico adultos que acuden a urgencias de traumatología en las 6 horas subsiguientes. El criterio de inclusión es la presencia de alcohol. Los pacientes positivos que aceptan la intervención son evaluados y aleatoriamente asignados a grupo de intervención mínima o breve. El seguimiento se realiza a los 3,6 y 12 meses. A los pacientes con una puntuación en el AUDIT sugerente de problemas graves de alcohol se les aconseja acudir a un centro especializado y se analizan aparte.

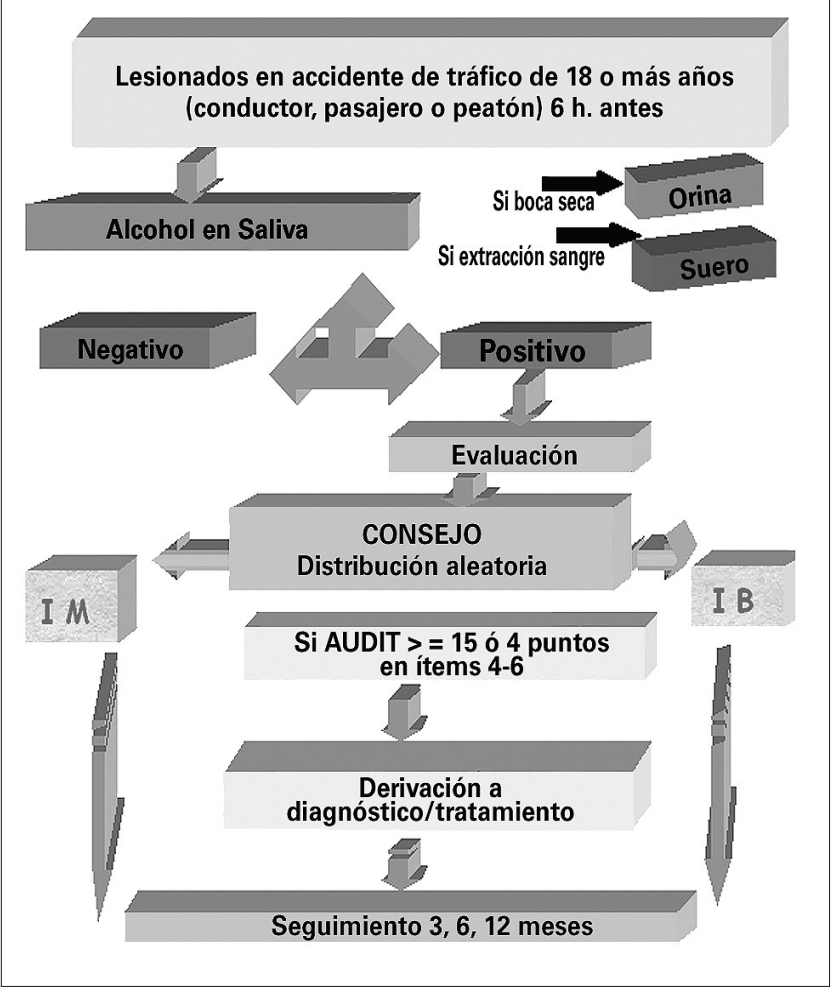

\section{Descripción de la muestra: perfil de los lesionados de tráfico con alcohol positivo.}

En los 12 meses del estudio (1 Julio 2001 a 28 febrero 2002, y 1 Julio 2002 a 30 Octubre 2002), se identificaron 811 lesionados de tráfico, de los que 709 resultaron elegibles (pacientes de 18 o más años, lesionados en un accidente de tráfico acontecido en las 6 horas precedentes).

La muestra obtenida representa el 8,1\% de los lesionados en AT adultos asistidos durante el periodo y no es representativa de la población atendida. Nuestra muestra es más joven (27 años de mediana) y contiene más varones $(69,3 \%)$ y conductores $(66,5 \%$ incluyendo un $28,7 \%$ de conductores de coche y un $35,7 \%$ de vehículos de 2 ruedas), probablemente porque, a pesar de mantener siempre abierta la captación de pacientes, se ha reforzado el cribado en fines de semana. La presencia de peatones es, probablemente por la misma razón, inapreciable $(5,1 \%)$. Los vehículos de 2 y 4 ruedas están igualmente representados, al sumarse conductores y pasajeros. 
Los pacientes con criterios de inclusión (709) fueron sobre todo reclutados en Julio $(31,3 \%)$ y de jueves a domingo, $(72,5 \%)$ y especialmente en el fin de semana (viernes a domingo, 55\%), con una distribución ligeramente superior $(58,2 \%)$ en el turno de noche (de 20,00 a 7,59). El horario más representado $(36,7 \%)$ es el de las 8 de la tarde hasta las 0,59 de la madrugada siguiente. Los lesionados que acuden en fin de semana son significativamente más jóvenes que los que acuden en días laborables.

La hora preferente del accidente es entre las 19,00 y las 20,59, y la hora de la asistencia, entre las 20,00 y las 21,59. La mediana del tiempo transcurrido entre accidente y asistencia es de una hora y media (moda: 1 hora).

El 15\% de los pacientes requiere hospitalización.

En el 98,2\% de los pacientes elegibles se realizó la determinación de alcohol en fluidos biológicos (95,2\% de casos, en saliva, el test de elección para el protocolo). Sólo 10 de los pacientes elegibles $(1,41 \%$ del total) rechazó el test.

De los 696 pacientes cribados, 93 (13,4\%: el $16,3 \%$ de los varones y el 6,9\% de mujeres) tenían un resultado positivo (alcoholemia de $\geq 0,2$ ), es decir, cumplían el criterio de inclusión en el estudio de efectividad.

\section{Descripción de los casos positivos a su entrada en el estudio}

Los pacientes con alcohol positivo presentan significativas diferencias con respecto a los negativos. Los positivos son sobre todo varones (83,9\%), se accidentan preferentemente $(73,1 \%)$ en horario nocturno y acuden más $(63,5 \%)$ durante el turno de noche (de 8 p.m. a 8 a.m.) y especialmente durante el fin de semana (el 76,3\% de casos positivos entre viernes y domingo), y quedan ingresados con mayor frecuencia $(29,0 \%)$. Aisladamente considerados, el sábado $(31,2 \%)$ y domingo $(34,4 \%)$ son los días con mayor porcentaje de casos positivos a expensas de las "noches" del viernes y sábado. Entre los pacientes positivos no hay más conductores que entre los negativos ni son significativamente más jóvenes.
De los 93 pacientes con alcoholemia positiva $(\geq 0,2)$, pudo intervenirse en $66(71,0 \%)$, que fueron aleatoriamente distribuidos en IB (30) e IM (36) y podían considerarse representativos del grupo de pacientes positivos. Las razones para la "no intervención" fueron sobre todo de tipo "logístico" (traslados prematuros, etc.). Pocos pacientes presentaron criterios de exclusión, siendo el rechazo el menos prevalente ( $2,2 \%$ de los positivos $-7,4 \%$ de los no intervenidos) y la intoxicación aguda no remitida antes del alta, el más prevalente.

2 pacientes intervenidos $(3,3 \%)$ se derivan para ulterior diagnóstico y eventual tratamiento por posible dependencia alcohólica.

La figura 2 ilustra numéricamente el desarrollo del algoritmo: captación, cribado, intervención y seguimiento de pacientes.

La evaluación, realizada en los pacientes con alcohol positivo como paso previo e inseparable de la intervención, permite la descripción de su perfil de consumidor al inicio el estudio (línea base).

Globalmente considerados, el 50\% (51.8\% de varones y $40 \%$ de mujeres) de los pacientes presentan un AUDIT positivo ( $\geq 8)$, con una mediana de 7.50 media de 7,65 (7,95, en los varones; 6,0 en las mujeres). Hemos usado el mismo punto de corte para ambos sexos, dada la escasa representación de mujeres y porque, en la presente muestra, ninguna puntúa por debajo del mismo.

La mayoría de pacientes incluidos son bebedores de riesgo, obteniendo la mayor parte de su puntuación $(60,8 \%)$ en el AUDIT en los 3 primeros ítems del cuestionario (frecuencia y cantidad del consumo, más episodios de consumo excesivo). Su media (para un máximo de 12 puntos, 4 por pregunta), es 4,65 y su mediana, de 4,00 (4,9- en los varones- y 3,2 -significativamente menor- en el caso de las mujeres).

Estudiamos separadamente el comportamiento de estos 3 primeros ítems, conocidos también como AUDIT-C (consumo), tanto por su peso específico como por ser éstos los que usamos en el seguimiento para valorar los cambios en el consumo. Catalogamos como "positivos", respecto al consumo, a quienes suman $\geq 5$ (varones) $0 \geq 4$ (mujeres) $^{32}$ puntos

Tabla 1. Características diferenciales entre pacientes positivos y negativos en la prueba de alcohol

\begin{tabular}{lccc}
\hline Variables & $\begin{array}{c}\text { Pacientes positivos } \\
(\mathbf{n = 9 3 )}\end{array}$ & $\begin{array}{c}\text { Pacientes negativos } \\
(\mathbf{n = 6 0 3 )}\end{array}$ \\
\hline Sexo (varón) & $78(83.9)$ & $401(66.5)$ & $=0.001$ \\
Ingreso hospitalario & $27(29.0)$ & $76(12.6)$ & $<0.001$ \\
Día de la asistencia (Viernes-Domingo) & $71(76.3)$ & $305(50.9)$ & $<0.001$ \\
Hora del accidente (8 p.m. to 7.59.am.) & $68(73.1)$ & $258(43.1)$ & $<0.001$ \\
Hora de la asistencia (8:00 p.m. and 7:59 a.m.) & $59(63.4)$ & $340(56.9)$ & $<0.001$
\end{tabular}


Fig. 2. Diagrama de la evolución del estudio. En los 12 meses del estudio (1 Julio 2001 a 28 febrero 2002- 1 Julio 2002- 31 Octubre 2002), se han detectado 811 lesionados (el 8,1\% de los accidentados adultos asistidos en el servicio de urgencias durante este periodo), algunos de los cuales hubo que desestimar, sobre todo por haberse lesionado más de 6 horas antes. El diagrama recoge la cadencia del estudio, detallando la información más relevante. El seguimiento es descrito según el porcentaje de pacientes controlados (37) dentro de la muestra intervenida en la que procedía ya algún control (48), y según el porcentaje de seguimientos que procedía dentro de cada cómputo establecido.

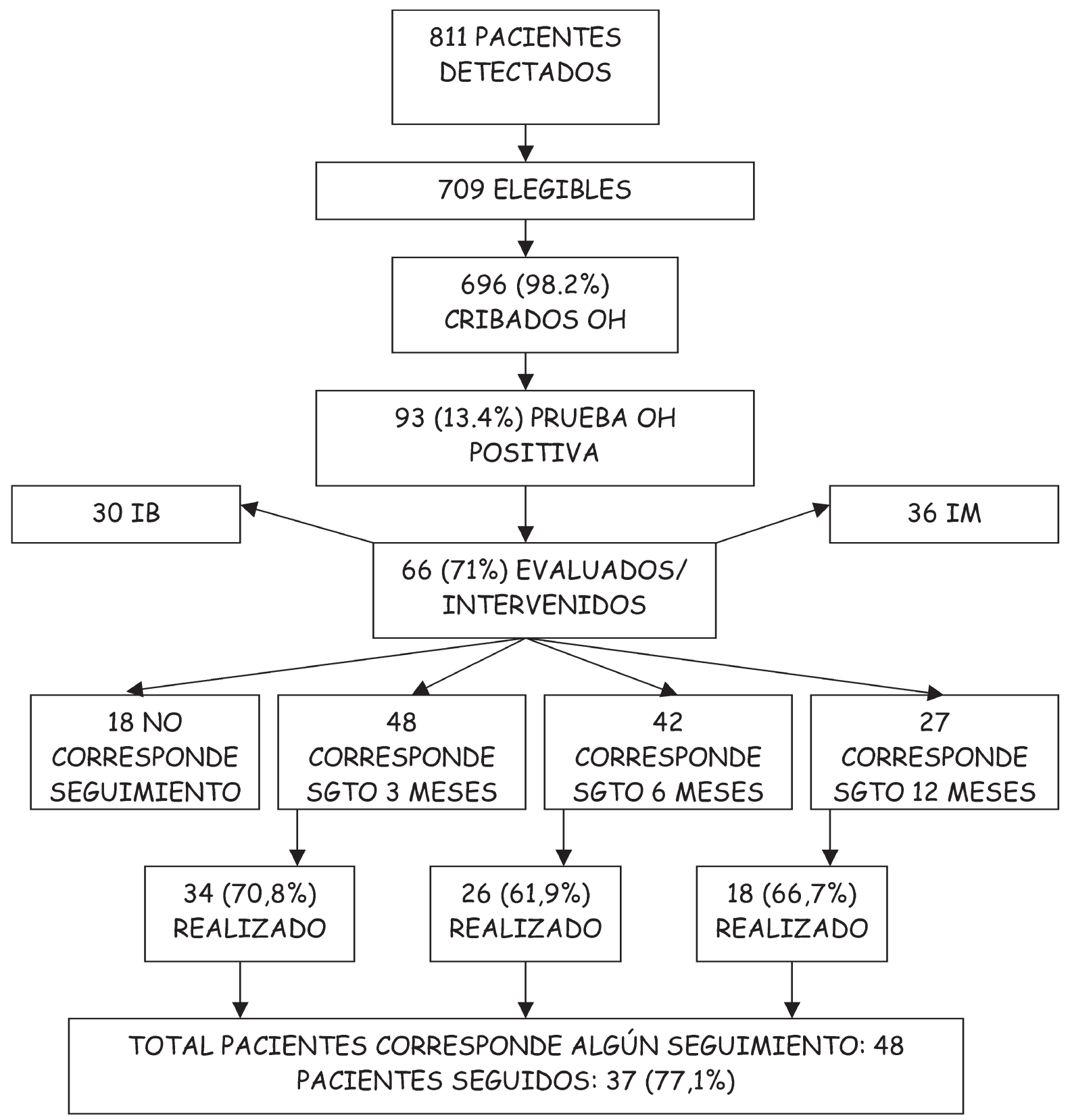

en estos ítems 1-2-3, también conocidos como AUDIT$\mathrm{C}^{33}$. El $51.5 \%$ de la muestra $(51,8 \%$ de los varones y el $50 \%$ de las mujeres con alcoholemia positiva) puntúan como bebedores de riesgo, de acuerdo con este criterio.

Los pacientes, aleatoriamente distribuidos tienen un porcentaje mayor de AUDIT positivos ( $\geq 8$ puntos) en el grupo de IB (NS).
En el inventario de actitudes, creencias y conductas, destaca el hecho de que el $69,9 \%$ de los pacientes reconoce haber tomado 3 o más bebidas en las 6 horas previas al accidente y que el $91,9 \%$ de ellos había bebido en las 3 horas previas. La mayor parte no tenía ni idea de la alcoholemia que podría haber tenido en el momento del accidente. El 59\% había tenido un accidente de tráfico (con o sin víctimas), el 42,1\% de ellos en el año anterior. 
En la escala de atribución a la lesión (de 1-7 puntos) la mediana para todos los lesionados positivos es de 3 puntos, y su disposición para el cambio (escala de 1-10 puntos) arroja un valor mediano de 7,0.

En conjunto, el seguimiento global alcanza el $77,1 \%$ en los pacientes a los que correspondía algún control. De los que entraron en el estudio un año antes, el 74,1\% de los pacientes algún seguimiento. De los que han podido ser evaluados al año, (el $66,7 \%$ ), el $75 \%$ tiene los tres controles (ver Fig. 2).

\section{RESULTADOS}

Los resultados pueden considerarse representativos de la muestra intervenida, dado que los dos grupos (IM e IB) son homogéneos en cuanto a edad, sexo, régimen de asistencia (ambulatoria u hospitalaria), papel en el tráfico, atribución a la lesión y preparación para el cambio. La única diferencia, no significativa, es relativa a la puntuación en el AUDIT, algo superior en el grupo IB.

A lo largo del seguimiento, reducen consumos todos los pacientes ( $>3 / 4$ partes en el mes 3 y 2/3 en los meses 6 y 12), con lo que se reduce también el porcentaje de aquellos que puntúan "positivo" en los ítems que exploran el consumo (AUDIT-C), sin diferencias significativas entre los porcentajes respectivos dentro de cada grupo de intervención (ver tabla 2), aunque a los tres meses la diferencia roce el límite de la significación estadística.

Tabla 2a. Porcentaje de pacientes que reducen consumo, medido por puntuación en AUDIT-C, según grupo de intervención

\begin{tabular}{lcccc}
\hline & $\begin{array}{c}\text { Total } \\
(\mathbf{N}=\mathbf{6 6})\end{array}$ & $\begin{array}{c}\text { IB } \\
(\mathbf{N}=\mathbf{3 0})\end{array}$ & $\begin{array}{c}\text { IM } \\
(\mathbf{N}=\mathbf{3 6})\end{array}$ & $\mathbf{P}$ \\
\hline Mes 3 & 79.4 & 94.1 & 64.7 & NS \\
Mes 6 & 65.4 & 64.3 & 66.7 & NS \\
Mes 12 & 66.7 & 69.2 & 60.0 & NS \\
\hline
\end{tabular}

Tabla 2b. Porcentaje de pacientes que puntúan "positivo" " en el AUDIT C en cada seguimiento, según grupo de intervención (IB ó IM)

\begin{tabular}{lcccc}
\hline & $\begin{array}{c}\text { Total } \\
(\mathbf{N =} \mathbf{6 6})\end{array}$ & $\begin{array}{c}\text { IB } \\
(\mathbf{N}=\mathbf{3 0})\end{array}$ & $\begin{array}{c}\text { IM } \\
(\mathbf{N}=\mathbf{3 6})\end{array}$ & $\mathbf{P}$ \\
\hline Basal & 51.5 & 56.7 & 47.2 & NS \\
Mes 3 & 14.7 & 23.5 & 5.9 & NS \\
Mes 6 & 19.2 & 28.6 & 8.3 & NS \\
Mes 12 & 27.8 & 38.5 & 0 & NS \\
\hline
\end{tabular}

${ }^{1}$ Punto de corte: $\geq 5$, para varones; $\geq 4$, para mujeres
A los 3 meses, el porcentaje de pacientes que puntúan positivo en el AUDIT $\mathrm{C}$ ha disminuido un $71,5 \%$; al año, la reducción sigue siendo importante (disminución del 51,8\% de casos positivos).

Al analizar el porcentaje de pacientes que reducen consumos, en función de su puntuación en el test AUDIT (AUDIT completo y AUDIT-C) a la entrada en el estudio, observamos diferencias significativas al año (la tabla 3 presenta la evolución según la puntuación basal en el AUDIT-C).

Tabla 3. Porcentaje de pacientes que reduce consumo, según AUDIT-C ${ }^{1}$ basal

\begin{tabular}{lcccc}
\hline & $\begin{array}{c}\text { Total } \\
\text { muestra } \\
\mathbf{N = 6 6}\end{array}$ & $\begin{array}{c}\text { Pacientes } \\
\text { AUDIT-C (+) } \\
\mathbf{N = 3 4}\end{array}$ & $\begin{array}{c}\text { AUDIT1 } \\
\mathbf{- C}(-) \\
\mathbf{N = 3 2}\end{array}$ & p \\
\hline Mes 3 & 79.4 & 94.7 & 60.0 & $<0.05$ \\
Mes 6 & 65.4 & 84.6 & 46.2 & NS \\
Mes 12 & 66.7 & 90.9 & 28.6 & $<0.05$ \\
\hline
\end{tabular}

${ }^{1}$ Punto de corte: $\geq 5$, para varones; $\geq 4$, para mujeres

Revisando individualmente cada ítem de consumo, observamos que la frecuencia de consumo (ítem 1) se reduce a lo largo del año de seguimiento: el consumo de 4 o más veces /semana pasa del 21,2\% (basal) al $6,1 \%$ (3 meses), al 9,1\% (6 meses) y al 4,5\% (12 meses). En cuanto a la cantidad (ítem 2), el consumo igual o superior a 3 consumiciones por día pasa del $72.8 \%$ basal, al 44,1\% (3 meses), al 35,6\% (6 meses) y al 44,5\% (12 meses). El consumo de 5 o más unidades diarias, pasa del 24,3\% basal al 5,9\% (3 meses), al 7,7\% (6 meses) y al 16,7\% (12 meses).

El antecedente de episodios de "atracones de consumo" (binge drinking) (6 o más consumiciones/ sesión de consumo) pasa del 63,6\% (basal), al 47,10\% (mes 3), al 34,60\% (mes 6) y al $27,80 \%$ (mes 12 ).

Fig. 3. Evolución del ítem $\mathbf{3}$ del cuestionario AUDIT. A lo largo de los 12 meses subsiguientes a la intervención, aumenta el porcentaje de pacientes que no ha incurrido en ningún "atracón" de alcohol en un 56,3\%.

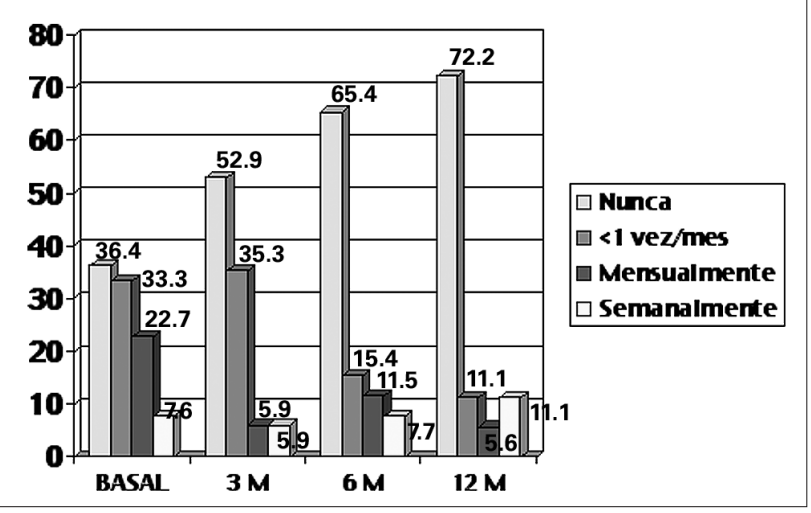


La magnitud de la reducción medida a partir de la media de puntuación basal en los ítems $1+2+3$, es de alrededor de 1,5 para el total de la muestra, manteniéndose al año.

La magnitud de la reducción es significativamente superior en aquellos pacientes que puntuaban positivo, tanto en el AUDIT completo (tabla 4) como en sus ítems de consumo o AUDIT-C (fig. 4), sin diferencias significativas por lo que respecta al grupo de intervención.

Tabla 4. Magnitud media de la reducción, según puntuación en el AUDIT total basal'

\begin{tabular}{lccrc}
\hline & $\begin{array}{c}\text { Total } \\
\text { muestra } \\
\mathbf{N = 6 6}\end{array}$ & $\begin{array}{c}\text { Pacientes } \\
\text { AUDIT (+) } \\
\mathbf{N = 3 3}\end{array}$ & $\begin{array}{c}\text { AUDIT } \\
(-\mathbf{~})\end{array}$ & $\mathbf{p}$ \\
$\mathbf{N = 3 3}$ & \\
\hline Mes 3 & 1.59 & 2.24 & 0.94 & $<0.05$ \\
Mes 6 & 1.54 & 2.64 & 0.73 & $<0.05$ \\
Mes 12 & 1.56 & 2.90 & -0.13 & $<0.05$ \\
\hline
\end{tabular}

punto de corte $\geq 8$

Fig. 4. Magnitud media de la reducción de consumo según la puntuación basal en el AUDIT-C.

La reducción de consumos es significativamente superior en los lesionados que puntuaban como consumidores de riesgo al entrar en el estudio ( $\geq 5$, los varones; $\geq 4$ las mujeres en los ítems de consumo o AUDIT-C).

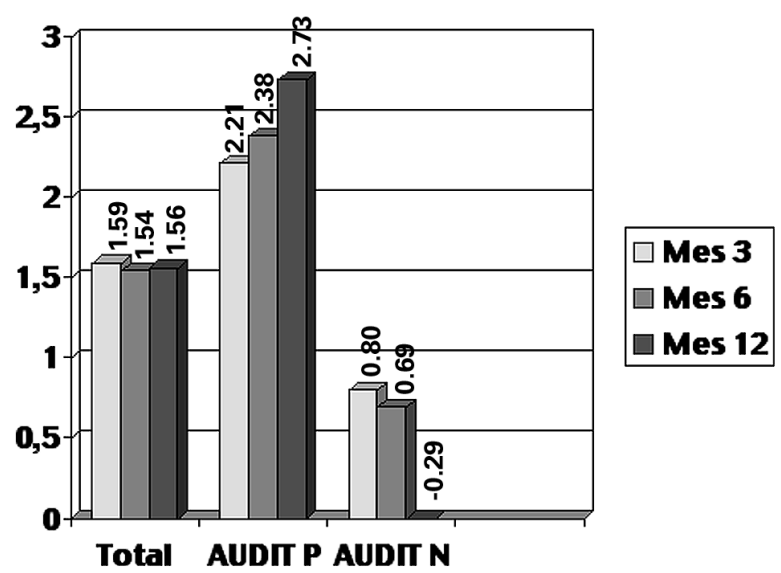

${ }^{1}$ Punto de corte: $\geq 5$, para varones; $\geq 4$, para mujeres

$\mathrm{P}<0,005$

Al año, había sufrido un nuevo accidente de tráfico el $16,7 \%$ de los pacientes seguidos (este antecedente figuraba en el $24,2 \%$ de los 66 pacientes intervenidos, para el año anterior a su inclusión en el estudio). Ninguno de los pacientes intervenidos manifestó haber sido multado por alcoholemia ilegal durante los 12 meses de seguimiento y el 5,6\% había sido multado por algún otro motivo.

\section{LIMITACIONES}

La muestra de pacientes es todavía demasiado reducida, especialmente al año y cuando la subdividimos en 2 grupos (IB - IM), para extraer conclusiones definitivas.

El seguimiento de pacientes $(77,1 \%$ de seguimiento global y $63,6 \%$ a los 12 meses), insuficiente para un estudio de eficacia, pero aceptable para un estudio de efectividad en un entorno de urgencias, es mejorable y se espera incrementarlo a lo largo del estudio.

La información recogida se limita a la información dada por el propio paciente, que puede considerarse fiable de acuerdo con estudios anteriores y porque la utilización de cuestionarios estandarizados (AUDIT) permite una mejor objetivación.

La muestra de lesionados incluidos en el estudio no es representativa de la población adulta de lesionados de tráfico que acude al hospital (por limitarse a los lesionado en AT en las 6 horas precedentes y porque el protocolo contemplaba cribar especialmente los lesionados de los fines de semana), aunque sí es representativa de los pacientes detectados. Las muestras respectivas de pacientes con alcohol positivo, pacientes a los que se ha hecho intervención (IB e IM), pacientes seguidos y perdidos son homogéneas en cuanto a las variables de la línea base.

\section{CONCLUSIONESY DISCUSIÓN}

- El ritmo de incorporación de pacientes ha sido inferior al esperado (709, en los 12 meses en que el estudio ha estado abierto).

Problemas de personal (reducción y conflictos en la plantilla) han afectado negativamente el ritmo de trabajo y el del estudio, tanto por lo que respecta a la obtención de la muestra como a la realización de la intervención en los pacientes con criterio de inclusión, a pesar de la contratación adicional de personal colaborador para las noches de fin de semana. Por otra parte, la composición de la muestra presenta un sesgo, al haber potenciado el cribado en las noches de fin de semana, las que tienen mayor prevalencia de AT con alcohol positivo, según indicó el estudio piloto ${ }^{34}$.

- Se analizó el alcohol en 696: 93 (13,4\%) dieron positivo (criterio de intervención).

El rechazo del cribado fue mínimo (1,4\%), como lo había sido en otro estudio realizado en un hospital general de Barcelona (1\%) con anterioridad ${ }^{35}$. El porcentaje de casos positivos, inferior al previsto en pacientes lesionados, es sin embargo similar al 
obtenido en el mencionado estudio ${ }^{35}$ en urgencias médicas y traumáticas. Aunque la prevalencia habitualmente referida por la literatura ronda el 30\%$40 \%$ 22,36,37, también se refieren porcentajes menores en caso de lesiones no graves (entre el 21,6 y $2,4 \%)^{38-40}$ y en atendidos en urgencias por lesiones no violentas $(10 \%$ en una muestra anterior de Barcelona $\left.{ }^{41}\right)$.

La prevalencia referida para los accidentados analizados por la policía " a pie de carretera", y por tanto inmediatamente posterior al accidente $(18,2 \%$ de casos positivos en el año $\left.2000^{10}\right)$, tampoco es muy superior a la nuestra.

Llama la atención la baja prevalencia de peatones, agrupados con los pasajeros como "no conductores", que no se corresponde ni con la prevalencia de peatones entre los lesionados de tráfico, ni con la frecuencia de intoxicados entre ellos, ya se trate de lesionados (en torno al $18 \%{ }^{11}$ ) y o fallecidos $\left(39,2 \%{ }^{\%}\right.$.

- Existen diferencias significativas entre los lesionados con y sin presencia de alcohol, siendo los positivos preferentemente varones que acuden durante el turno de noche, en fin de semana y precisan ingreso por su gravedad.

Este perfil coincide, salvo por lo que concierne a la edad, con el referido por la literatura internacional, lo que avala el valor predictivo ${ }^{42,35}$ de determinadas variables (edad, sexo masculino, gravedad de las lesiones, horario nocturno y fin de semana) y obligaría a extremar el cribado de problemas de alcohol en los sujetos acogidos a este perfil.

- 66 recibieron intervención (71\% de los positivos), siendo aleatoriamente distribuidos entre IM e IB.

Nuestro porcentaje de pacientes "AUDIT- positivos" es muy alto $(50 \%)$, más que el habitualmente referido por la literatura internacional y nacional. En atención primaria, se estima que alrededor del $15-20 \%$ son bebedores de riesgo (en la población general, alrededor del 10\%). No tenemos información acerca de la prevalencia de bebedores de riesgo en lesionados españoles. Sin embargo, los estudios de Cherpitel ${ }^{40}$ refieren una prevalencia de "AUDIT-positivos" del $30 \%$ en lesionados, frente al $22 \%$ en pacientes de urgencias no traumáticas. Recientemente (Smith et al., 2003 ${ }^{43}$ se ha comunicado una prevalencia de AUDITs positivos del $95,4 \%$ en lesionados faciales que hubieran consumido 8 o más unidades (de $8 \mathrm{~g}$ = $64 \mathrm{~g}$ ) antes de la lesión. En nuestro caso, estamos explorando pacientes lesionados y con presencia de alcohol, lo que podría explicar una mayor probabilidad de presentar consumos de riesgo y problemas con el alcohol.
Existen evidencias contradictorias acerca de una correlación entre la presencia de alcohol en pruebas biológicas y una puntuación positiva en el test AUDIT ${ }^{40,42,44}$. Maio et al ${ }^{42}$ refieren una prevalencia ponderada de abuso/dependencia de alcohol en el $22,5 \%$ en pacientes lesionados atendidos en urgencias, presentando una alcoholemia positiva el 55\% de los que tienen un diagnóstico de problemas de alcohol, frente al $6 \%$ de los casos con diagnóstico negativo. Cherpitel ${ }^{40}$, en cambio, descarta que la presencia de alcohol en aire espirado sea un buen predictor de dependencia en pacientes traumáticos. En nuestra muestra, si bien la mitad de pacientes con alcohol positivo tienen también un AUDIT positivo ( $\geq 8)$, no tenemos apenas pacientes que superen los límites establecidos en el protocolo para derivación por posible dependencia alcohólica, de acuerdo con las recomendaciones de la literatura existente ${ }^{29,45,46,47}$ y nuestro valor medio en el AUDIT $(7,65)$ no es superior al referido por otros autores $(12,8)^{25}$.

Dado que, a lo largo del seguimiento, se han utilizado de nuevo los 3 primeros ítems del AUDIT para comprobar la evolución del consumo, se ha explorado el peso de estos primeros ítems dentro del test completo (contribución del 60,8\%) y elegido un punto de corte (el de $\geq 5$ para los varones $y \geq 4$ para las mujeres), para esta versión abreviada o AUDIT$\mathrm{C}^{33}$, de entre los propuestos por la literatura, por ser éste el que mejor correlaciona con el diagnóstico en el AUDIT completo, dentro de nuestra muestra. Se estima necesario un próximo estudio de la efectividad del AUDIT-C en lesionados.

- El 37,1\% de los pacientes no ve relación alguna entre el AT y su consumo de alcohol, aunque un tercio considera que ha contribuido en gran parte o totalmente al mismo (mediana 3,00 en una escala de 1-7).

Estos datos son análogos a los obtenidos por Sommers et al. ${ }^{48}$, aunque su muestra se polarizaba en "nada" y "en gran parte" o "totalmente", mientras que en la nuestra la atribución importante está menos presente. Si tenemos en cuenta que nuestra muestra incluye un tercio de no conductores, no es tan sorprendente que un porcentaje análogo no vea relación entre el accidente y su consumo de alcohol.

- Casi la mitad de pacientes se sienten dispuestos a cambiar su conducta con el alcohol (mediana de 7 en una escala de 1-10). Sólo un 16,1\% no cree que deba cambiar nada.

Nosotros no encontramos, por ahora, la correlación referida por Sommers ${ }^{48}$ entre -atribución de la lesión al alcohol- preparación para el cambio.

- Los 66 pacientes positivos, distribuidos aleatoriamente, han recibido una IB (30) o una IM (36). 
El grupo de IM, que recibió un simple consejo hace las veces de control. No tenemos un grupo de control "puro" (no intervención = no evaluación ni consejo) por motivos éticos y por considerar que la eficacia de las intervenciones breves en general ya ha sido demostrada en bebedores excesivos no dependientes. Otros autores también han prescindido de un control con "no intervención", tanto en España ${ }^{49-50}$ como en otros países ${ }^{23,51-54}$. En nuestro caso, se trataría más bien de comprobar la efectividad de la IB comparada con la IM.

Los pacientes aceptaron muy bien la intervención y su distribución aleatoria en IM e IB generó muestras homogéneas y comparables.

- 37 pacientes de los 48 a los que correspondía algún seguimiento $(71,1 \%)$ han sido seguidos, lo que representa el $75 \%$ de los que entraron en el estudio hace 1 año.

El seguimiento ha sido efectuado por un colaborador que no conocía el grupo de adscripción (ciego), como exige la fiabilidad del estudio y mediante entrevista telefónica, considerada tan fiable como la personal ${ }^{55}$. El porcentaje de seguimiento alcanzado por el momento, aunque no ideal (se estima que en estudios de eficacia no debería ser inferior al $80 \%{ }^{58}$, es aceptable. La mayor parte de estudios no alcanzan el $80 \%$ propuesto, incluyendo algunos tan importantes como el de la $\mathrm{OMS}^{19}$, aunque se comunican porcentajes de hasta el $83 \%$ de seguimientos en pacientes lesionados ${ }^{25,43}$. La mayor parte de pérdidas se deben a la no localización del paciente (cambios teléfono, cambio de domicilio...).

Los pacientes seguidos y perdidos constituyen muestras homogéneas, por lo que cabría asumir que su evolución ha podido ser la misma que en los entrevistados. A diferencia del estudio de Macken$z^{2}{ }^{57}$, aquí no podemos inferir que los no localizados hayan evolucionado peor que los que no acuden, porque nadie "acude".

- Todos los lesionados en AT con presencia de alcohol que recibieron intervención redujeron su consumo, especialmente a los 3 meses, pero manteniéndose al año.

Esta observación es concordante con los hallazgos de otros autores $38,46,25,58,59$ y plantea la cuestión, aún abierta ${ }^{58}$, de si el propio AT constituye una experiencia aversiva suficiente para explicar la reducción de consumos. En cualquier caso, el evento negativo podría explicar acaso otra observación reiterada: que la reducción es más manifiesta a los 3 meses.
- Destaca el porcentaje de reducción global de los episodios de "atracones" (binge drinking), los más asociados a la accidentalidad.

Al igual que Sommers ${ }^{38,58}$, observamos una continua disminución de los "atracones" desde la evaluación basal hasta el año de seguimiento, sin diferencias entre grupos. Si el ítem 3 del AUDIT es considerado como el mejor indicador de consumo de riesgo en poblaciones no clínicas hasta el punto de haberlo propuesto para un cribado abreviado y rápido ${ }^{33}$, la modificación de este parámetro ha de ser entendida como un claro beneficio, especialmente entre lesionados. Un año después de la intervención, se ha doblado el porcentaje de quienes no incurren en episodios de consumo excesivo (0 puntos en el ítem) y se reduce a la mitad el porcentaje de quienes presentan episodios regulares (mensuales o semanales $=2$ ó 3 puntos en el ítem). Aisladamente considerado, el porcentaje de bebedores copiosos con frecuencia semanal no varía apenas a lo largo del seguimiento, pero el exiguo número de pacientes que incurren en esa categoría (2) no permite un análisis diferenciado.

- El estudio de efectividad no arroja diferencias significativas entre grupo de intervención: $>75 \%$ de los lesionados ha disminuido su consumo a los 3 meses y el $>60 \%$, al año. De un $51.5 \%$ de lesionados con AUDIT-C positivos, al inicio, se pasa a un $14.7 \%$ a los 3 meses y a un $27.8 \%$ al año. La magnitud de la reducción oscila entre una media de 1,59, a los 3 meses y 1,56, al año.

El pequeño tamaño de nuestra muestra hace prematura cualquier conclusión. Sin embargo, cabría la posibilidad de que la IB no aportara beneficio adicional con respecto al consumo de alcohol, globalmente considerado ${ }^{38,59}$, pero sí respecto a las pautas de mayor riesgo y a la accidentalidad derivada ${ }^{24,25}$.

- Existen diferencias significativas de reducción de consumos, según el grupo de bebedor al que pertenece el paciente de acuerdo con su puntuación en el AUDIT. Los lesionados que puntúan por encima del nivel diagnóstico de riesgo, tanto en el AUDIT total como en el AUDIT-C (ítems consumo) reducen su consumo significativamente más (mayor \% de pacientes y mayor magnitud de la reducción) que los pacientes sin consumo de riesgo.

Esta significativa diferencia es importante porque nos revela que reducen sobre todo los que realmente lo necesitan. Dado que el criterio de inclusión en el estudio es la presencia de alcohol al ser admitido en un servicio de urgencias por AT, existe la posibilidad de que incluyamos a bebedores ligeros que aquel día han bebido, así como de que excluyamos a bebedores con problemas de alcohol que ese día no 
han bebido. Por eso es especialmente relevante ajustar el contenido de la intervención al resultado del AUDIT (refuerzo positivo de los bebedores prudentes, consejo de reducción en los excesivos, derivación de los posibles dependientes), insistiendo en la relación entre alcohol - riesgo viario. El riesgo más elevado de sufrir lesiones en relación con el alcohol se da entre jóvenes que toman 5 o más consumiciones alcohólicas sólo dos veces al año ${ }^{3}$.

- Al cabo de 1 año, la prevalencia de AT (con o sin víctimas) referidos por los pacientes para los últimos 12 meses ha disminuido un 60,3\% respecto a los referidos a la entrada en el estudio para el año precedente.

Es prematuro comparar esta reducción entre ambos grupos por la reducida muestra de pacientes que cumplen 1 año en el estudio. La literatura refiere tanto una significativa reducción en todos los grupos terapéuticos ${ }^{25}$, como una reducción significativamente superior en quienes reciben una IB frente a controles ${ }^{22}$.

Hemos presentado la descripción y análisis de los datos recogidos hasta 31 de Octubre de 2002, dentro de un estudio que sigue incluyendo pacientes hasta el 30 de Abril de 2003. Se trata pues de resultados provisionales, que sin embargo avanzan una información de interés.

El perfil de los lesionados con presencia de alcohol nos indica el grupo poblacional sobre el que debemos concentrar nuestros esfuerzos de identificación e intervención: varones que se lesionan de gravedad y acuden en las noches de fines de semana. La mitad de sujetos con alcohol positivo es además identificado como bebedor de riesgo por un test AUDIT. Tras la intervención, se reduce el consumo y en especial el antecedente de atracones. La magnitud de la reducción es significativamente superior en quienes tenían un AUDIT positivo. No se evidencian aún diferencias significativas por grupo de intervención.

Sólo al final del estudio podremos comprobar la hipótesis de efectividad. Un valor añadido del estudio será, en cualquier caso, haber favorecido la concienciación de los profesionales de urgencias de traumatología acerca del elemento "alcohol" como factor de riesgo para las lesiones y acerca del potencial preventivo de quienes están en la "primera línea".

\section{AGRADECIMIENTOS}

Agradecemos sinceramente la colaboración de todo el personal de enfermería del Servicio de Urgencias del Hospital de Traumatología y Rehabilitación y muy especialmente la esforzada participación de los profesionales que se formaron y aplicaron tanto el cribado como la evaluación-intervención: Raquel Benedit, Jordi Capilla, Fanny Galeano, Carolina Gómez, Miguel López, Helena Quesada, Tania Rodríguez-Martos, y Aurora Vilella.

\section{BIBLIOGRAFÍA}

(1) González Luque, JC: Conducción de vehículos. Consumo de alcohol en los jóvenes. En: Gil et al.Subdirección Grl de Epidemiología, Promoción y Educación para la Salud.: Alcohol y Juventud 1995. Madrid, Ministerio de Sanidad y Consumo. 1996. (pp.: 87-104).

(2) Cía MT, Arévalo JM, Ardanaz E, Barricarte A, Cheuca P, Serrano S.: Nivel de alcohol y riesgo de lesión por accidente de tráfico en Tudela (Navarra). Gac Sanit 1996; 10: 55-61.

(3) Cherpitel CJ, Tam T, Midanik L, Caetano, R, Greenfield T. Alcohol and non-fatal injury in the U.S. general population: a risk function analysis. Accid Anal Prev 1995; 27 (5): 651-661.

(4) Cherpitel, CJ. Drinking patterns and drinking problems associated with injury status in emergency room admissions. Alcoholism Clin Exp Res 1988; 12 (1): 105110.

(5) MacDonald S, Wells S, Giesbrecht N, Cherpitel C J. Demographic and substance use factors related to violent and accidental injuries: results from an emergency room study. Drug and Alcohol Dependence 1999; 55: 53-61.

(6) Rivara, FP; Jurkovich GJ; Gurney, JG; Seguin, D; Fligner, $\mathrm{CL}$; Ries, R; Raisys, VA; Copass, M.: The magnitude of acute and chronic alcohol abuse in trauma patients. Arch Surg 1993; 128 (8): 907-913.

(7) Charalambous MP. Alcohol and the accident and emergency department. A current review. Alcohol \& Alcoholism 2002; 37 (4): 307-312.

(8) Rivara, FP; Koepsell, TD; Jurkovich, GJ; Gurney, JG; Sodeberg, R.: The effects of alcohol abuse on readmission for trauma. JAMA 1993; 270 (16): 19621964.

(9) Instituto de Toxicología. Memoria. Análisis Toxicológico de las Muertes en Accidentes de Tráfico Memoria. Año 2000. Ministerio de Justicia.

(10) Dirección General de Tráfico. Boletín Informativo del Consejo Superior de Tráfico y Seguridad de la Circulación Vial. SEGURIDAD VIAL N 103. Diciembre 2001. Ministerio del Interior, España.

(11) Dirección General de Tráfico. Investigaciones para la Seguridad Vial. Ministerio del Interior, España. 2000 (pp: 141-144; 249-251).

(12) Plasencia A: Bases epidemiológicas para las políticas de prevención de los accidentes de tráfico relacionados con el alcohol en España. Adicciones 1999; 11 (3):229236. 
(13) Evans L. The fraction of traffic fatalities attributable to alcohol. Accid Anal Prev 1990; 22 (6): 587-602.

(14) Girasek DC, Gielen AC, Smith GS. Alcohol's contribution to fatal injuries: A report on public perceptions. Ann Emmerg Med 2002, 39 (6): 622-630.

(15) Longabaugh, R; Minugh, PA; Nirenberg, TD; Clifford, PR; Becker, B; Woolard, R.: Injury as a motivator to reduce drinking. Academy of Emergency Medicine 1995; 2: 817-825.

(16) Dyehouse JM, Sommers MS: Brief intervention as an advanced practice strategy for seriously injured victims of multiple trauma. AACN Clinical Isues 1995; 6 (1): 5362.

(17) Gentilello LM, Donovan DM, Dunn C, Rivara FP. Alcohol interventions in level 1 trauma centers: Current practice, future directions. JAMA 1995; 274: 1043-1048.

(18) Welte J; Perry P; Longabaugh R; Clifford PR: An outcome evaluation of a hospital-based early intervention program. Addiction 1998; 93 (4): 573-581.

(19) Babor TF, Grant M (eds.) Programme on Substance Abuse. Project on identification and management of alcohol-related problems. Report on Phase II: a randomized clinical trial of brief intervention in primary health care. World Health Organization, Geneva, 1992.

(20) Bien, Th.; Miller, W.R.; Tonigan, J.S.: Brief interventions for alcohol problems: a review. Addiction 1993; 88: 315336

(21) Wilk Al; Jensen NM; Havighurst TC: Meta-analysis of randomized control trials assessing brief interventions in heavy alcohol drinkers. J Gen Int Med 1997; 12: 274283.

(22) Gentilello, LM, Rivara FP, Donovan DM, Jurkovich GJ, Daranciang E, Dunn $\mathrm{CH}$, Villaveces A, Copass M, Ries RR. Alcohol Interventions in a trauma center as a means of reducing the risk of injury recurrence. Annals of Surgery 1999; 230 (4): 473-483.

(23) Wright S, Moran L, Meyrick M, O'Connor R, Touquet R. Intervention by an alcohol health worker in an accident and emergency department. Alcohol \& Alcoholism 1998; 33: 651-656.

(24) Monti PM;Colby SSM; Barnett NP; Spirito A; Rohsenow DJ; Myers $M$; Woolard R; Lewander W: Brief intervention for harm reduction with alcohol-positive older adolescents in a hospital emergency department. J Consult Clin Psychol 1999; 67 (6): 989-994.

(25) Longabaugh R, Woolard RF, Nirenberg TD, Minugh AP, Becker B, Clifford PR, Carty K, Sapadeo F \& Gogineni A. Evaluating the effects of a brief motivational intervention for injured drinkers in the emergency department. J Stu Alc 2001; 62: 806-816.

(26) Ballesteros J: ¿Es eficaz la intervención breve en bebedores a riesgo en el ámbito de la atención primaria? Una revisión sistemática de estudios españoles. Trastornos adictivos 2000; 2 (1): 32-40.

(27) Ballesteros J., Ariño J, González-Pinto A, Querejeta I. Eficacia del consejo médico para la reducción del consumo excesivo de alcohol. Metaanálisis de estudios españoles en atención primaria. Gaceta Sanitaria 2003; 17 (2): 116-122.

(28) Saunders JB, Aasland OG. WHO collaborative project on the detection and treatment of persons with harmful alcohol consumption. Report on phase I: development of a screening instrument. World Health Organization, Geneva, 1987.

(29) Rubio G, Bermejo J, Caballero MC, Santodomingo J. Validación de la prueba para la identificación de trastornos por uso de alcohol (AUDIT) en atención primaria. Rev Clín Esp 1998; 198: 11-14.

(30) Longabaugh, R; Minugh, PA; Nirenberg, TD; Clifford, PR; Becker, B; Woolard, R.: Injury as a motivator to reduce drinking. Academy of Emergency Medicine 1995; 2: 817-825.

(31) D'Onofrio G, Bernstein E, Bernstein J, Woolard R, Brewer Ph A, Craig SA et al.: Patients with alcohol problems in the emergency department, part 2: Intervention and referral. Academic Emerg Med 1998; 5: 1210-1217.

(32) Gual A, Segura L, Contel M, Haether N, Colom J. AUDIT-3 and AUDIT-4: Effectiveness of two short forms of the alcohol use disorders identification test. Alcohol \& Alcoholism 2002; 37 (6):591-596.

(33) Bush K, Kivlahan DR, McDonell MB, Fihn SD, Bradley KA.: The AUDIT alcohol consumption questions (AUDITC): an effective brief screening test for problem drinking. Arch Intern Med 1998; 158: 1789-1795.

(34) Rodríguez-Martos A, Plasencia A, Escayola M, Martí J, Torralba LI. Intervención breve en accidentados con alcoholemia positiva desde un centro de traumatología. Adicciones 2001; 13 (4): 371-383.

(35) Cherpitel CJ, Parés A, Rodés J, Rosovsky H: Validity of self-reported alcohol consumption in the emergency room: data from the United States. Mexico and Spain. J Stud Alcohol 1992; 53:203-207.

(36) Ankney RN, Vizza J, Coil JA, Kurek S, DeFrehn R, Shomo $\mathrm{H}$. Cofactors of alcohol-related trauma at a rural trauma center. Am J Emerg Med 1998; 16 (3): 228-231.

(37) Cunningham RM, Maio RF, Hill EM, Zink BJ. The effects of alcohol on head injury in the motor vehicle crash victim. Alcohol \& Alcoholism 2002; 37 (3): 236-240.

(38) Sommers MS, Dyehouse JM, Howe, SR. Brief intervention trial in injured patients: Issues and results. Presented at the XXVII Annual Epidemiology Symposium of the Kettil Bruun Society for Social and Epidemiological research in Alcohol. Toronto (Canada), May-June 2001

(39) Lillis RP, Good R, States JD. Post-treatment driving records of injured drivers. In: Thirty-ninth Annual Proceedings, Association for the Advancement of Automotive Medicine, 1996; 39: 79-87.

(40) Cherpitel CJ. Screening for alcohol problems in the emergency department. Ann Emerg Med. 1995; 26: 158-166.

(41) Cherpitel C.J. Alcohol and injuries resulting from violence: a review of emergency room studies. Addiction 1994; 89: 157-165. 
(42) Maio RF, Waller PF, Blow FC, Hill EM, Singer KM.: Alcohol Abuse / Dependence in Motor Vehicle Crash Victims presenting to the Emergency Department. Academic Emergency Medicine, 1997;4 (4):256-262.

(43) Smith AJ, Hodgson RJ, Bridgeman K, Sheperd JP. A randomized controlled trial of a brief intervention after alcohol-related facial injury. Addiction 2003; 98 (1): 4352

(44) Clifford PR, Longabaugh R. Minugh PA, Nirenberg T, Becker B, Woolard R. Utilization of saliva alcohol screens in hospital emergency department to differenciate problematic and non-problematic drinkers. Alc Clin Exp Res 1994; 18: 429.

(45) Babor TF, Higgins- Biddle JC, Saunders JB, Monteiro M. AUDIT The Alcohol Use Identification Test. Guidelines for Use in Primary Care.WHO/MSB/01.6a. World Health Organization, Geneva, 2001.

(46) Contel M, Gual A, Colom J. Test para la identificación de trastornos por uso de alcohol (AUDIT): traducción y validación del AUDIT al catalán y castellano. Adcciones 1999; 11: 337-347.

(47) Sommers MS, Dyehouse JM, Howe SR, Weeks A, Russell AC-: Using brief interventions following alcoholrelated motor vehicle crashes to reduce drinking and further injury. Proceedings of the $17^{\text {th }}$ Conference on Alcohol, Drugs and Traffic Safety, Stockholm, 20-25 May 2000: pp 73.

(48) Sommers MS, Dyehouse JM, Howe SR, Lemmink J, Davis K, McCarthy M, Russell AC: Attribution of Injury to Alcohol Involvement in young adults seriously injured in alcohol-related motor vehicle crashes. Am J Crit Care 2000; 9 (1): 28-35.

(49) Altisent, R.; Córdoba, R.; Delgado, MT; Pico, MV.; Melús, E.; Aranguren, F. et al.: Estudio multicéntrico sobre la eficacia del consejo para la prevención del alcoholismo en Atención Primaria EMPA. Medicina Clínica (Barc.) 1997; 109: 121-124.

(50) Córdoba R, Delgado MT, Pico V, Altisent R, Fores D, Monreal A, Frisas O, López del Val, A: Effectiveness of brief intervention on non-dependent alcohol drinkers (EIBAL): a Spanish Multi-centre study. Family Practice 1998; 15 (6): 562-568.

(51) Kuchipudi V, Hobein K, Flickinger A et al.: failure of a 2hour motivational intervention to alter recurrent drinking behaviour in alcoholics with gastrointestinal disease. J Stud Alc 1990; 51: 356-359.

(52) Romesljö A, Anderson L, Barrner $\mathrm{H}$ et al.: A randomized study of secondary prevention of early stage problem drinkers in primary helath care. Br J Addict 1989; 84: 1319-1327.

(53) Maheswaran R, Beevers M, Gareth D: Effectiveness of advice to reduce alcohol consumption in hypertensive patients. Hypertension 1992; 19: 79-84.

(54) Nilssen O: The Tromso study: identification of and a controlled intervention on a population of early-stage risk drinkers. Prev Med 1991; 20: 518-528.

(55) Greenfield TK, Midanik LT, Rogers JD. Effects of telephone versus face-to-face interview models on reports of alcohol consumption. Addiction 2000; 95(2): 277-284.

(56) Kahan M, Wilson L, Becker L: Effectiveness of physician-based interventions with problem drinkers: a review. Can Med Assoc J 1995; 152: 851-859.

(57) Mackenzie A. et al. The characteristics of alcoholics frequently lost to follow-up. J Stud Alc 1987; 48 (2): 119-123.

(58) Sommers MS, Dyehouse JM, Howe SR: Binge drinking, sensible drinking, and abstinence after alcohol-related vehicular crashes: The role of intervention versus screening. 45 $5^{\text {th }}$ Annual Proceedings Association for the Advancement of Automotive Medicine. Sept. 24-26 2001 (pp: 317-328).

(59) Moyer A, Finney JW, Swearingen CE, Vergun P.: Brief interventions for alcohol problems: a meta-analytic review of controlled investigations in treatmentseeking and non-treatment - seeking populations. Addiction 2002; 279-292. 